\title{
Correspondence
}

\section{A complication of external jugular vein catheterization in children}

\section{To the Editor:}

Double- and triple-lumen catheters for central venous placement have recently become popular for use in the Operating Room and Intensive Care Unit. They are particularly useful in cases where central venous pressure (CVP) monitoring must be carried out simultaneously with fluid or drug infusions. I would like to report two recent instances of problems with these catheters in paediatric patients.

\section{Case \#1}

A $52 \mathrm{~kg}, 15$-year-old, healthy girl was scheduled for spinal fusion and Harrington instrumentation for idiopathic scoliosis. After an uncomplicated anaesthetic induction, the patient was placed in the Trendelenberg position for insertion of a CVP catheter. The double-lumen catheter chosen was 5.0 French size (16-gauge equivalent) and $15 \mathrm{~cm}$ in length (Cook Canada Ltd., 16-151 Esna Park Dr., Markham, Ont. L3R 3B1. Product \#C-DLMS01J-LSC*). The patient's right external jugular vein was prominent and easily cannulated with a 20 -gauge intravenous catheter. The 0.018 inch $(0.46 \mathrm{~mm})$ spring-wound "J"-wire was inserted and passed to an appropriate length with a slight "twirling" motion to negotiate the bend where the external jugular vein meets the subclavian and internal jugular veins to form the brachiocephalic vein. ${ }^{1}$ The intravenous catheter was removed over the guide wire and the double-lumen catheter introduced against some resistance (even after enlarging the skin incision). The catheter was inserted to about $13 \mathrm{~cm}$, and easy return of blood through the proximal lumen was noted. Considerable resistance was felt during attempted removal of the guide wire from the catheter. The central core of the wire suddenly broke, leaving the distal portion attached only by the fragile spring wire, which unraveled rapidly as the proximal fragment was pulled (Figure 1). The break occurred within the "tail" of the distal lumen, so that it was out of sight but still outside the patient. Fortunately we were able to place a haemostat tightly around the catheter at skin level and remove the catheter and distal

*Lot \#6504.75100. This lot was distributed only to our hospital. portion of the wire as one unit. There was no apparent injury to the patient and the case was completed with no other mishaps and with a satisfactory surgical and anaesthetic result. Examination of the guide wire showed no evidence of manufacturing fault or weakness, and there was no kink in the wire at the site of breakage. The case was reported to the manufacturer and to the Bureau of Medical Devices. The manufacturer responded promptly to our concerns but was unable to provide a definitive explanation for the incident.

\section{Case \#2}

A $14 \mathrm{~kg}$, 14-year-old girl with cerebral palsy was prepared for a Luque procedure for spinal fusion. She had progressive kyphoscoliosis which interfered with positioning in her wheelchair, but had no evidence of respiratory embarrassment. Anaesthesia was induced and the patient was positioned for CVP monitoring line insertion. The catheter was again a double-lumen type of the same diameter, but was $12 \mathrm{~cm}$ long (Cook Canada \#C-DLM-501J-RSC). The catheter was placed via the right external jugular vein without problem, except that the guide wire could be felt sticking within the catheter during removal. Slow and gentle traction removed the wire without damage. The catheter worked well throughout the case. There was easy blood withdrawal from the proximal lumen and a typical central venous pressure waveform was transduced from the distal lumen. Aspiration of blood from the distal lumen was only intermittently

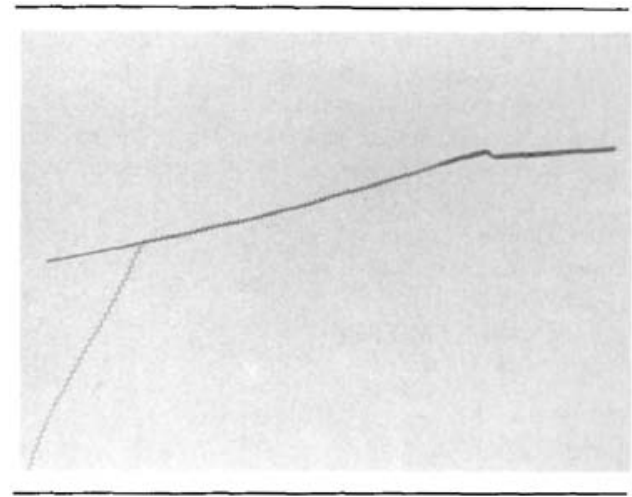

FIGURE I Case 1. Unraveled extemal spring component of guide wire following fracture of central core component. 


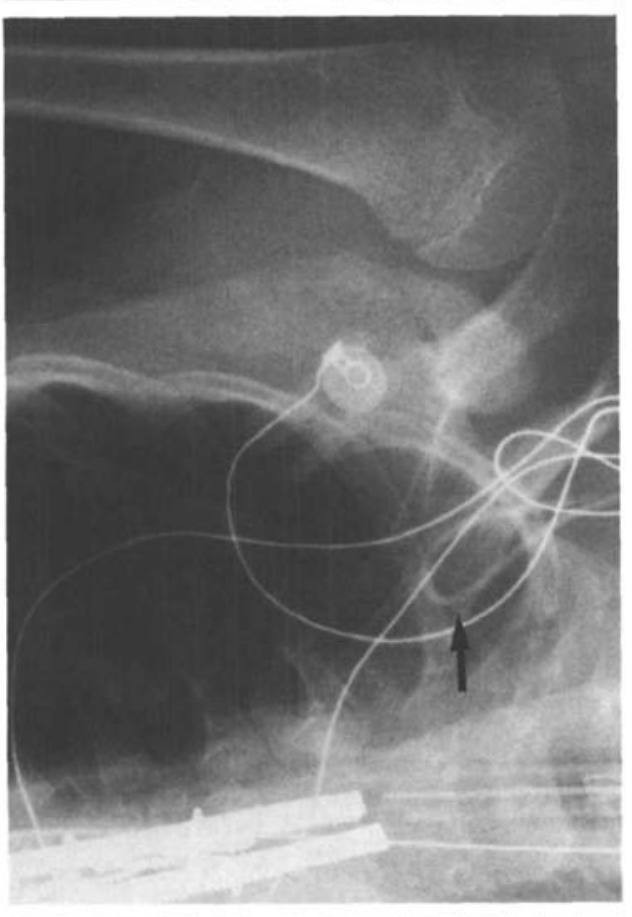

FIOURE 2 Case 2 . Chest $x$-ray taken in Intensive Care Unit.

possible, however. After the procedure, a chest $x$-ray was taken in the ICU, and the CVP catheter was seen to be bent, with the tip apparently pointing back up the external jugular vein (Figure 2), but there was no clinical or radiological indication that it was extravascular. The catheter was removed without injury to the patient.

\section{Discussion}

Any physician with experience in central venous catheterization can relate anecdotes of catheters that did not go where they were supposed to; the problems described may not seem particularly surprising. However, the above two cases may be related, in that a similar bend in the guide wire and catheter in the first case may have been the cause of the sticking and subsequent breaking of the wire. Most physicians who treat adults are familiar with the standard 0.035 -inch spring-wound " $\mathrm{l}$ "-wire. Compared with that, the centre portion of the 0.018 -inch " $I$ "-wire is extremely flexible, and the tips are even more so. This flexibility is considered a safety feature, as there have been many reports of perforations of the right atrium or major veins by central venous pressure monitoring lines or guide wires. ${ }^{2-4}$ However, this flexibility will also allow the wire to follow unexpected routes, or even to be "led" by the catheter itself, particularly when the approach to the superior vena cava is via the more tortuous external jugular system. In addition, a "16-gauge equivalent" double-Jumen catheter has lumens that are only equivalent to 20 -gauge and are thus more liable to interfere with removal of the wire.

Our experience suggests caution in the use of thin, flexible guide wires for central venous catheterization by the external jugular route. Great care must be taken with 0.018 -inch guide wires if there is any resistance to removal, and simultaneous removal of both the catheter and the wire may be advisable. Most anaesthetists do not routinely $x$-ray patients following central line insertion in the operating room, but any difficulty in removing the guide wire or in aspirating blood should be an indication to do so. However, my colleagues and I have used these catheters for internal jugular and femoral vein catheterization many times without difficulty, and we have, in general, been very satisfied with their performance and with the response of the manufacturer to our concerns.

G. Allen Finley MD FRCPC

Department of Anaesthesia

Dalhousie University

Izaak Walton Killam Children's Hospital

Halifax, Nova Scotia B3J 3G9

\section{REFERENCES}

1 Clemente $C D$. Anatomy - A Regional Atlas of the Human Body, Philadelphia: Lea \& Febiger, 1975 (Fig. 437).

2 Collier PE, Ryan JJ, Diamond DL. Cardiac tamponade from central venous catheters. Report of a case and review of the English literature. Angiology 1984; 35: 595-600.

3 Perforation of the Heart and Vessel by Central Venous Catheters Inserted for CVP Monitoring and TPN Therapy. (Medical Devices Alert No. 79), Health Protection Branch, Dept. of National Health and Welfare, Ottawa, March 7, 1986.

4 Jay $A W L$, Kehler $C H$. Editorial: heart perforation by central venous catheters. Can I Anaesth 1987; 34: 333-5

\section{Combined spinal-epidural needle (CSEN)}

To the Editor:

We would like to describe a new device we have developed to facilitate the use of the combined spinal- 